\title{
Composición florística y distribución de los bosques ribereños subtropicales andinos del Río Lules, Tucumán, Argentina
}

\author{
Martín G. Sirombra ${ }^{1}$ \& Leticia M. Mesa ${ }^{2}$ \\ 1. Facultad de Ciencias Naturales e Instituto Miguel Lillo, Universidad Nacional de Tucumán, Miguel Lillo 255, \\ Argentina; sirombra@gmail.com \\ 2. Fundación Miguel Lillo, Miguel Lillo 255, Tucumán, Argentina; leticiamesa@yahoo.com.ar
}

Recibido 12-V-2009. Corregido 12-VII-2009. Aceptado 25-VIII-2009.

\begin{abstract}
Floristic composition and distribution of the Andean subtropical riparian forests of Lules River, Tucuman, Argentina. We studied the floristic composition and distribution of the riparian forest of two hydrographical systems in a subtropical Andean region. Using uni and multivariate techniques, we tested the hypotheses that a differentiable riparian forest exists, composed by native vegetation typical of the Yungas phytogeographical province, and that the distribution of vegetation varied significantly with geomorphologic characteristics. Parallel transects along the water courses were used to collect presence-absence data of vegetation in eleven sites. Detrended Correspondence Analysis defined a group of common riparian species for the studied area (Solanum riparium, Phenax laevigatus, Tipuana tipu, Cestrum parqui, Carica quercifolia, Acacia macracantha, Celtis iguanaea, Juglans australis, Pisoniella arborescens, Baccharis salicifolia, Cinnamomum porphyrium and Eugenia uniflora) and identified two reference sites. The distribution of the riparian vegetation varied significantly with the geomorphic characteristics along the studied sites. Riparian habitats were composed by native and exotic species. A distinct riparian flora, different in structure and function from adjacent terrestrial vegetation, could not be identified. Riparian species were similar to the adjacent terrestrial strata. These species would not be limited by the proximity to the river. Anthropogenic impacts were important factors regulating the introduction and increase of exotic vegetation. The lack of regulation of some activities in the zone could cause serious problems in the integrity of this ecosystem. Rev. Biol. Trop. 58 (1): 499-510. Epub 2010 March 01.
\end{abstract}

Key words: Argentina, mountain streams, riparian forest, subtropical climate, Yungas.

Las zonas ribereñas representan la interfase entre hábitats terrestres y acuáticos (Naiman et al. 1993). Esta propiedad les otorga atributos físicos y químicos específicos, propiedades bióticas y procesos de flujo de materia y energía, con interacciones únicas entre los sistemas ecológicos adyacentes (Naiman et al. 1988, Risser 1993). Diversos procesos fluviales, tales como crecidas y deposición del suelo aluvial, modelan la zona ribereña. Estos factores determinan, a su vez, la formación de una flora característica, típicamente diferente en estructura y función de la vegetación terrestre adyacente (Gregory et al. 1991, Naiman et al. 1993, Tang \& Montgomery 1995, Prach \&
Straskrabová 1996, Naiman \& Décamps 1997). $\mathrm{Su}$ función como proveedoras de alimento para organismos acuáticos, moderadoras de la temperatura del agua, filtradoras de nutrientes y contaminantes (Naiman et al. 1993), estabilizadoras de los bancos de los ríos (Barling \& Moore 1994, Hood \& Naiman 2000) y como corredores para el movimiento de organismos (Naiman \& Décamps 1997), las convierten en zonas de vital importancia para el mantenimiento de la biodiversidad.

La región andina del noroeste de Argentina, con aproximadamente $4.500 .000 \mathrm{~km}^{2}$, es muy heterogénea a nivel de paisaje y comprende una amplia extensión de bosque húmedo 
hiperdiverso denominado Yungas. Esta área incluye las cabeceras de importantes cuencas, cuyos ríos atraviesan valles intermontanos utilizados para la agricultura y pasturas, en frágiles condiciones de pendiente y suelo.

Diversas actividades humanas amenazan la integridad de la zona ribereña del noroeste de Argentina (NOA). El avance de la frontera agrícola, la introducción de ganado que ramonea la vegetación hasta el límite del cauce y compacta el suelo por pisoteo, los asentamientos humanos y la extracción de agua, son algunas de las actividades que afectan la funcionalidad de estos ecosistemas creando, a su vez, ambientes propicios para el establecimiento de vegetación exótica (Richardson et al. 2007).

Las consecuencias de la introducción de especies exóticas pueden variar, desde provocar un ligero cambio en la composición de especies hasta la extinción de plantas nativas, generando esto último, una profunda modificación del ecosistema natural (Parker \& Reichard 1997). Los sistemas ribereños son propensos a la invasión por especies exóticas debido a que poseen una elevada relación área-borde, lo que provee de múltiples puntos de entrada para propágulos de especies exóticas (Ede \& Hunt 2009).

Estas amenazas sumadas a la falta de conocimiento de la estructura y composición de la comunidad ribereña de los ríos del NOA vuelven necesario el estudio inmediato de este tipo de ecosistema. Las hipótesis planteadas fueron que el bosque ribereño se encuentra compuesto de vegetación nativa típica de la región biogeográfica de Yungas, y que la distribución de la vegetación varía en relación con ciertas características geomorfológicas.

\section{MATERIALES Y MÉTODOS}

Área de estudio: La cuenca Río Lules (26의 $\left.\mathrm{S} 65^{\circ} 45^{\prime} \mathrm{W}\right)$ se encuentra ubicada en el Noroeste de Argentina (NOA, Tucumán) (Mesa 2006). El sistema de ordenamiento de sus ríos revela una alta jerarquización (orden siete) y un alto grado de ramificación, con un elevado número de ríos de bajo orden que fluyen hacia el colector principal o hacia ríos de orden superior (Mesa 2006) (Fig. 1). Las alturas máximas se encuentran en la región occidental de la cuenca $(4.488 \mathrm{~m})$, y disminuyen hacia la zona pedemontana (zona oriental, $408 \mathrm{~m})$. Sobre la Sierra de San Javier (26 $39^{\circ}$ 'S $\left.65^{\circ} 17^{\prime} \mathrm{O}\right)$, límite oriental de la cuenca Río Lules, se encuentra un sistema de ríos de bajo orden (1 y 2) que se infiltran al llegar a la zona de llanura. Esta superficie comprende un área protegida "Parque Sierra de San Javier" de 14.000ha aproximadamente, administrada por la Universidad Nacional de Tucumán (Fig. 1). La altitud de la Sierra de San Javier varía entre los 600 y $1.900 \mathrm{~m}$.

Las dos áreas de estudio se hallan comprendidas dentro de la región biogeográfica de Yungas. Esta provincia fitogeográfica, extendida entre las coordenadas $22^{\circ}$ y $28^{\circ}$ de latitud Sur, y con un área de 3.900 .000 ha aproximadamente, representa una de las regiones más biodiversas de Argentina (Brown 2000). El clima subtropical monzónico caracteriza a la zona de estudio, determinando la ocurrencia del $80 \%$ de la precipitación anual entre los meses de Noviembre a Abril. La precipitación media anual es de $1141 \mathrm{~mm}$, presentándose valores máximos y mínimos de esta variable en enero $(226 \mathrm{~mm})$ y agosto $(11.8 \mathrm{~mm})$ respectivamente (Datos aportados por la estación meteorológica 'Obispo Colombres' de Tucumán período 1961-1990). Este factor confiere a la vegetación una fisonomía particular, característica de este tipo de clima.

Muestreos: Para la caracterización de las dos áreas seleccionadas se definieron once sitios de muestreo: ocho ubicados en la cuenca Río Lules: Anfama (ANF), Siambón (SIA), Las Juntas 1 (LJ1), Las Juntas 2 (LJ2), Potrerillo (POT), San Javier (SJ), Tablas (TAB), Membrillo (MEM), y tres situados sobre la sierra de San Javier: Río Muerto (MUE), Las Cañitas (CAÑ) y Tafí (TAF). San Javier se encuentra ubicado en la vertiente occidental de esta Sierra (Fig. 1). La cabecera y tramos superiores de los ríos Muerto, Las Cañitas y Tafí se encuentran protegidos por el Parque Sierra de San Javier. 


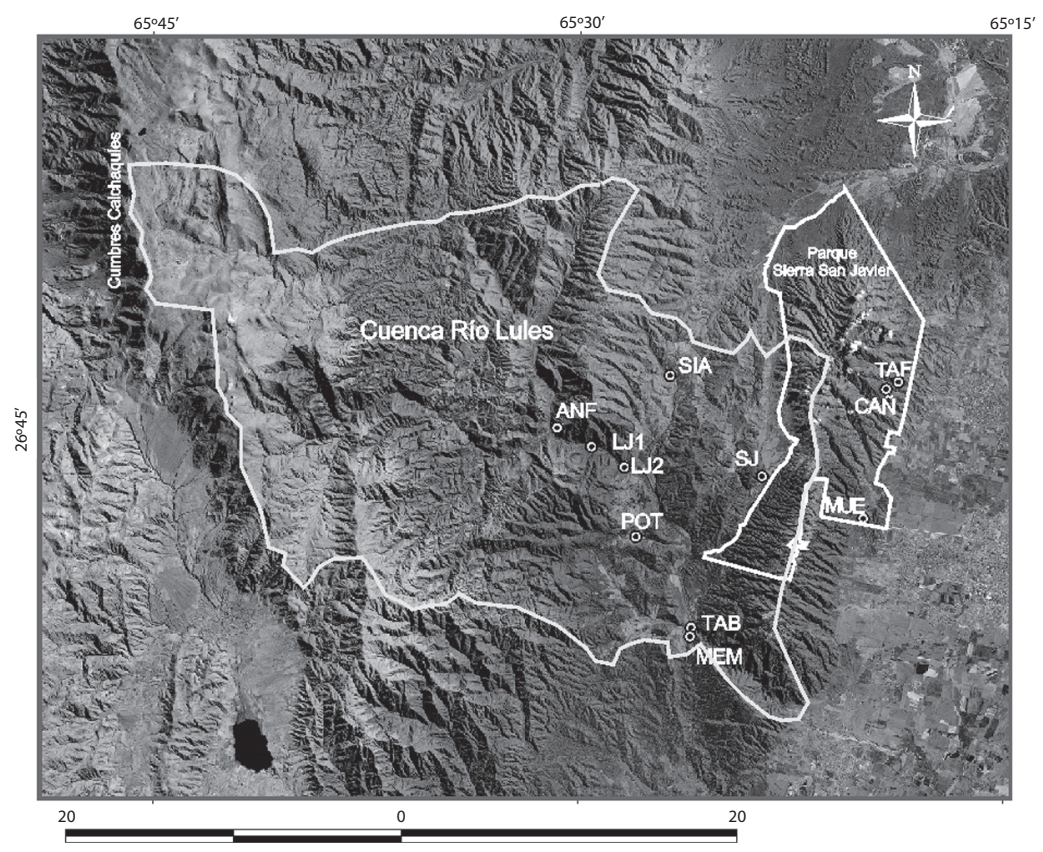

Fig. 1. Imagen Landsat de los dos sistemas hidrográficos estudiados (Sierra de San Javier y cuenca Río Lules) mostrando los once sitios de muestreo. Referencias de sitios: ANF= anfama, SIA= Siambón, LJ1= Las Juntas 1, LJ2= Las Juntas 2 , POT=Potrerillo , SJ= San Javier, TAB=Tablas, MEM= Membrillo, MUE= Río Muerto, CAÑ $=$ Las Cañitas, TAF= Tafí.

Fig. 1. Landsat image of the two studied hydrographic systems (Sierra de San Javier and Lules river basin) showing the eleven sampled sites. Sites references: ANF= Anfama, SIA= Siambón, LJ1= Las Juntas 1, LJ2= Las Juntas 2 , POT=Potrerillo , $\mathrm{SJ}=$ San Javier, $\mathrm{TAB}=$ Tablas, $\mathrm{MEM}=$ Membrillo, $\mathrm{MUE}=$ Río Muerto, $\mathrm{CAN}=\mathrm{Las}$ Cañitas, $\mathrm{TAF}=\mathrm{Tafí}$.

Aproximadamente a la altura de la cota $900 \mathrm{~m}$, abandonan el área protegida y discurren hacia la llanura tucumana.

Los muestreos de la vegetación ribereña se llevaron a cabo durante los meses de primavera, verano, otoño e invierno de los años 2003 al 2008. Para los propósitos de este estudio, se adaptó la metodología propuesta por Munné et al. (1998) para ríos de montaña. Para ubicar cada sitio de muestreo se marcó al azar, un punto de referencia inicial en el centro del lecho del río. La unidad de muestreo consistió en una línea recta de $50 \mathrm{~m}$ de longitud dispuesta sobre la primera franja de vegetación leñosa, en ambos márgenes, siguiendo el canal fluvial en sentido contrario a la corriente y considerando como referencia el punto central marcado previamente. Debido a las características geomorfológicas de las riberas de los tramos montañosos de estos ríos, el ancho del bosque ribereño y consecuentemente el de la unidad de muestreo, fue variable. En la mayoría de los sitios de muestreo el corredor ribereño fue angosto, por lo que el propio borde del bosque se consideró como el límite de la llanura de inundación. Referencias auxiliares como la presencia de pilas de detritos dejadas por flujos de inundación previos, la posición de las terrazas fluviales, la presencia de plantas indicadoras y/o la topografía de las orillas, facilitaron la identificación y delimitación de la zona de influencia del agua del río en las riberas adyacentes cuando los límites fueron difusos. Dentro del área ribereña, se incluyeron individuos arbóreos a partir de $30 \mathrm{~cm}$ de perímetro a la altura del pecho (PAP). Se registraron datos de presencia-ausencia de especies ribereñas de árboles, arbustos y lianas. Para la determinación taxonómica de las especies vegetales se siguió la propuesta de Zuloaga \& 
Morrone (2009). Además, se midió la altura del talud (bankfull), ancho del lecho seco, ancho del lecho húmedo respectivo a cada sitio. Para la determinación del orden del río se utilizó una imagen satelital Landsat, escala 1:75.000 (método de Strahler 1964, Mesa 2006).

Análisis estadístico: Se calcularon medidas de riqueza específica (como número de especies) de la vegetación ribereña de cada sitio de muestreo como primera descripción de la comunidad (Magurran 1989). Los datos de presencia-ausencia de especies fueron explorados mediante un Análisis de Correspondencia Detendenciado (ACD, programa PC-ORD ${ }^{\circledR}$, McCune \& Mefford 1999). ACD fue elegido debido a que esta técnica indirecta efectúa una ordenación teniendo en cuenta sólo los datos de la comunidad, permitiendo una expresión de gradientes comunitarios puros (Øklan 1996). Los sitios (representados por puntos sobre el diagrama de ordenación) se hallan distribuidos a lo largo de los ejes con base a su similitud en la composición de especies, con distancias en el diagrama equivalentes a las diferencias en composición taxonómica. Para realizar este análisis, las especies presentes en un solo sitio de muestreo fueron eliminadas para reducir la variabilidad causada por las especies raras
(Tausch et al. 1995). Una prueba de Mantel (999 permutaciones, Manly 1997) fue usada para probar la relación entre las coordenadas de los sitios (scores) sobre los dos primeros ejes del ACD con las siguientes variables: altitud del sitio, riqueza total, número total de especies exóticas, número de orden de río, altura media del talud (bankfull), ancho medio del lecho seco y ancho medio lecho húmedo. Si la hipótesis nula es correcta (no existe correlación entre los dos grupos de valores de distancia), los valores de la prueba estadística para los datos observados podría no estar en la cola superior (5\%) de los valores de distribución calculados al azar a partir de los datos permutados (Manly 1997).

\section{RESULTADOS}

En el Cuadro 1 se encuentran descritas las características físicas de los once sitios de muestreo. Los sitios comprenden un rango altitudinal de $425 \mathrm{~m}$ y el número de orden de río varió entre 2 y 7 . Asimismo, un total de 74 especies fueron definidas dentro de las dos zonas de estudio: 54 arbóreas, 14 arbustivas, 3 apoyantes, 2 cañas y 1 trepadora. Del total, $21 \%$ fueron especies exóticas, mientras que el

CUADRO 1

Características fisicas de los once sitios de muestreo ubicados en orden decreciente de altitud

TABLE 1

Physic characteristics of the eleven sampled sites in decreasing order of altitude

\begin{tabular}{|c|c|c|c|c|c|}
\hline Sitios & $\begin{array}{l}\text { Altitud } \\
\text { (m.s.n.m) }\end{array}$ & $\begin{array}{l}\text { Orden } \\
\text { de río }\end{array}$ & $\begin{array}{l}\text { Altura del talud } \\
\text { (bankfull) (m) }\end{array}$ & $\begin{array}{c}\text { Ancho del } \\
\text { lecho seco (m) }\end{array}$ & $\begin{array}{l}\text { Ancho del lecho } \\
\text { húmedo }(\mathrm{m})\end{array}$ \\
\hline Anfama & 1105 & 6 & 6.2 & 13.6 & 14.7 \\
\hline Siambón & 1080 & 5 & 2.5 & 11 & 18.2 \\
\hline Las Juntas 1 & 1070 & 7 & 1.2 & 21 & 14.1 \\
\hline Las Juntas 2 & 925 & 7 & 1.6 & 12.3 & 35 \\
\hline Potrerillo & 915 & 5 & 1.3 & 9.1 & 21.4 \\
\hline San Javier & 860 & 6 & 3 & 14.5 & 53.5 \\
\hline Cañas & 803 & 2 & 2.3 & 15.7 & 14.97 \\
\hline Muerto & 800 & 2 & 2.8 & 46.4 & 24.5 \\
\hline Tafí & 794 & 2 & 9 & 36 & 4.44 \\
\hline Tablas & 680 & 6 & 2 & 3 & 2.5 \\
\hline Membrillo & 680 & 5 & 1.6 & 7.5 & 2 \\
\hline
\end{tabular}


$79 \%$ restante correspondió a vegetación nativa (Anexo 1).

Los valores de riqueza de especies exóticas y nativas por sitio de muestreo, en orden decreciente de altitud, se presentan en la Fig. 2. Los 4 sitios ubicados sobre ambas vertientes de la Sierra San Javier presentaron una mayor riqueza de especies ribereñas ( $>27$ nativas, entre 3 y 11 exóticas) en comparación con con aquellos relativos a la cuenca Río Lules ( $<24$ nativas, entre 1 y 2 exóticas). Del total de especies exóticas, 5 arbóreas (Morus alba, Gleditsia triacanthos, Ligustrim lucidum, Psidium guayaba, Acacia macracantha), 1 arbusto (Pyracantha angustifolia) y una especie de caña (Arundo donax) son reconocidas como invasoras.

Análisis multivariado: Los dos primeros ejes del ACD explicaron la mayor parte de variación (48\%) existente en los datos (variación total=1.27). El eje 1 separó a los sitios de acuerdo a su posición geográfica: los ubicados sobre ambas vertientes de la Sierra de San Javier en el extremo inicial del eje 1 vs. los sitios pertenecientes a la cuenca Río Lules en el otro extremo (Fig. 3A). Asimismo, la altitud diferenció a los sitios sobre el eje 2: Anf y LJ1, sitios de mayor altitud, sobre el extremo inicial del eje 2 vs. Tab y Pot (sitios de menor altitud) en el extremo terminal (Fig. 3A). Ocho especies (Urera baccifera, Trema micrantha, Eucaliptus grandis, Arundo donax, Heliocarpus popayanensis, Pinus taeda., Psidium guajava y Psychotria carthagenensis) caracterizaron a los sitios de ambas vertientes de la Sierra de San Javier, mientras que seis especies (Zanthoxilum fagara, Parapiptadenia excelsa, Myrsine laetevirens, Verbesina suncho, Acacia praecox y Ruprechtia laxiflora) distinguieron a los sitios localizados en la cuenca Río Lules (Fig. 3B). Sobre el eje 2, Schinus bumelioides, Ricinus communis, Urera caracasana, Boehmeria caudata, y Allophylus edulis, caracterizaron a Tab y Pot, mientras que Cedrela lilloi y Terminalia triflora caracterizaron a LJ1 y Anf (Fig. 3B). Un grupo de especies nativas ubicadas en el centro del ACD (Solanum riparium, Phenax laevigatus, Tipuana tipu, Cestrum parqui, Carica quercifolia, Acacia macracantha, Celtis iguanaea, Juglans australis, Pisoniella

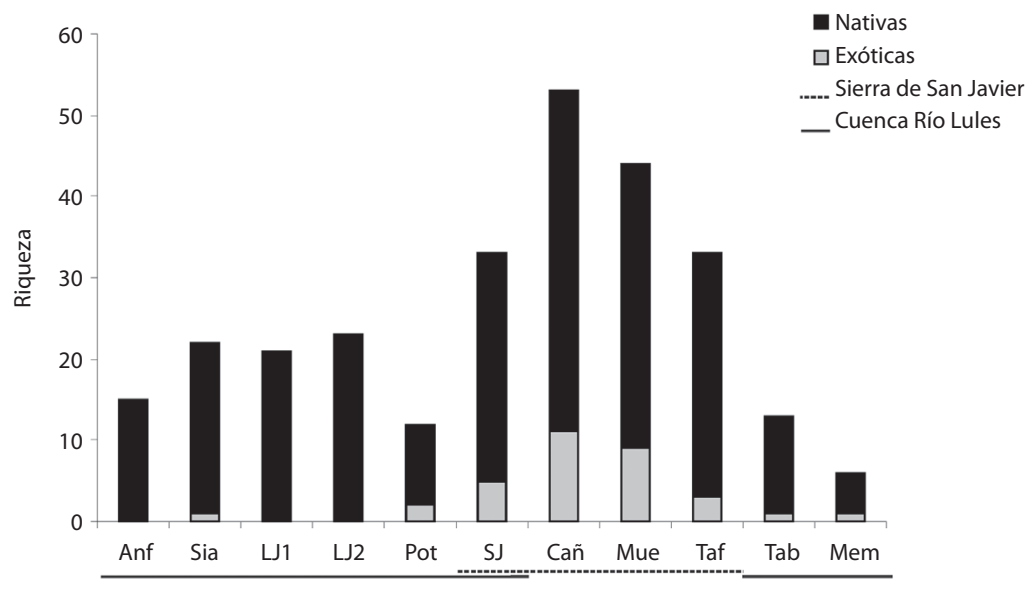

Fig. 2. Riqueza (como número de especies) de especies nativas y exóticas en los once sitios de muestreo. Los sitios se encuentran ubicados en orden decreciente de altitud. Anf, Sia, LJ1, LJ2, Pot, SJ, Tab, Mem=cuenca Río Lules; SJ, Cañ, Mue y Taf $=$ Sierra de San Javier. Anf $=$ Anfama, Sia $=$ Siambón, LJ1= Las Juntas 1, LJ2= Las Juntas 2, Pot=Potrerillo, SJ= San Javier, $\mathrm{Tab}=$ Tablas, Mem $=$ Membrillo, Mue $=$ Río Muerto, Cañ $=$ Las Cañitas, $\mathrm{TAF}=$ Tafí.

Fig. 2. Richness (as a total number of species) of native and exotic species of the eleven sampled sites. Sampled sites are situated in decreasing order of altitude: Anf, Sia, LJ1, LJ2, Pot, SJ, Tab, Mem =Río Lules basin; SJ, Cañ, Mue y Taf=San Javier hills. Anf $=$ Anfama, $\mathrm{Sia}=$ Siambón, LJ1 $=$ Las Juntas $1, \mathrm{LJ} 2=$ Las Juntas 2, Pot $=$ Potrerillo , SJ $=$ San Javier, Tab= Tablas, Mem= Membrillo, Mue= Río Muerto, Cañ $=$ Las Cañitas, TAF= Tafí. 

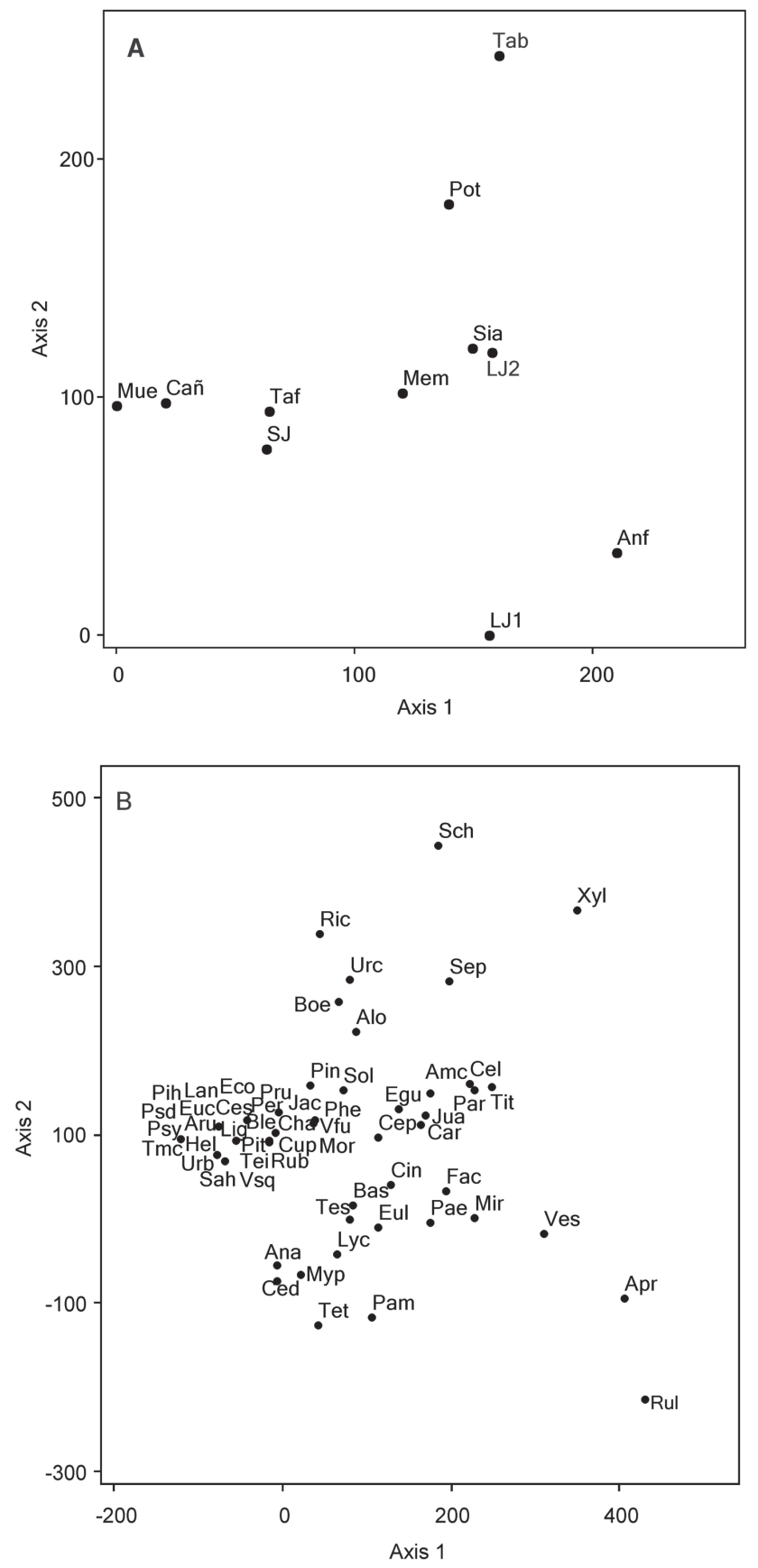

Fig. 3. Análisis de Correspondencia Detendenciado de la vegetación ribereña de los once sitios de muestreo. (A) Ordenación de sitios: Mue=Muerto; Cañ=Las Cañitas; Taf=Tafi; SJ=San Javier; Tab Tablas; Pot=Potrerillo; Mem=Membrillo; Sia=Siambón; LJ2=Las Juntas 2; Anf=Anfama; LJ1=Las Juntas 1. (B) Ordenación de especies. Ver Anexo 1 para referencias.

Fig. 3. Detendence Correspondence Analysis of riparian vegetation of the eleven sampled sites. (A) Sites ordination: Mue=Muerto; Cañ=Las Cañitas; Taf=Tafí; SJ=San Javier; Tab=Tablas; Pot=Potrerillo; Mem=Membrillo; Sia=Siambón; LJ2=Las Juntas 2; Anf=Anfama; LJ1= Las Juntas 1. (B) Species ordination. For references see Appendix 1 
CUADRO 2

Análisis de correlación entre las coordenadas de los sitios (scores) sobre los ejes 1 y 2 del Análisis de Correspondencia Detendenciado y siete variables (Prueba de Mantel, ${ }^{*}<<0.05$, $* * p<0.01$ )

TABLE 2

Results of correlation analysis between sites scores of axis 1 and 2 of Detendence Correspondence Analysis and seven variables (Mantel's test, ${ }^{*} p<0.05, * * p<0.01$ ).

$\begin{array}{lcccc}\text { Variables } & \text { Eje } 1 & p & \text { Eje } 2 & p \\ \text { Altura } & 0.50 & 0.06 & -0.54 & 0.04 * \\ \text { Riqueza } & -0.83 & 0.00^{* *} & -0.23 & 0.26 \\ \text { Total de exóticas } & -0.92 & 0.00^{* *} & -0.02 & 0.48 \\ \text { Orden de río } & 0.80 & 0.002^{* *} & -0.05 & 0.56 \\ \text { Altura del talud } & -0.41 & 0.13 & -0.09 & 0.50 \\ \text { Ancho del lecho seco } & -0.57 & 0.04 * & -0.34 & 0.14 \\ \text { Ancho del lecho húmedo } & 0.52 & 0.04^{*} & 0.16 & 0.30\end{array}$

arborescens, Baccharis salicifolia, Cinnamomum porphyrium y Eugenia uniflora) fueron comunes a todos los sitios muestreados.

Las coordenadas de los sitios (scores) sobre el primer eje del ordenamiento se relacionaron positivamente con el ancho del lecho húmedo y el orden de río (Prueba de Mantel, $\mathrm{R}>0.50$, $\mathrm{n}=10, \mathrm{p}<0.05$ ), $\mathrm{y}$ negativamente con la riqueza de especies, total de exóticas y ancho medio del lecho húmedo (Prueba de Mantel, R> -0.50 , $\mathrm{n}=10, \mathrm{p}<0.05$ ). Por otra parte, variables tales como la altura se relacionaron negativamente con el eje 2 (Prueba de Mantel, $\mathrm{R}>-0.50, \mathrm{n}=10$, $\mathrm{p}<0.05$ ) (Cuadro 2).

\section{DISCUSIÓN}

Este estudio diferenció un bosque ribereño cuya composición de especies se corresponde directamente con la vegetación terrestre adyacente. Todas las especies ribereñas identificadas ya fueron citadas como especies típicas de la región biogeográfica de Yungas (Morales et al. 1995). Esto podría indicar que este tipo de vegetación no depende de las fluctuaciones hidrométricas fluviales (Placci 1995, Neiff 2004) y que no están limitadas por la cercanía al río.

En contraposición con la primera hipótesis, este trabajo encontró que la vegetación ribereña de la provincia biogeográfica de
Yungas se encuentra compuesta por especies nativas y exóticas. Algunas de estas últimas son invasoras, condición que representa una situación de peligro para ecosistemas, hábitats u otras especies, con potencial daño económico y/o ambiental. Las consecuencias negativas de la introducción de exóticas son evidentes en algunos sectores de la cuenca Río Lules, donde la invasión de especies tales como Acacia macracantha y Gleditsia triacanthos ha reemplazado a la vegetación nativa, produciendo notables cambios fisonómicos y estructurales en el bosque ribereño el que paisajísticamente, se asemeja más a un bosque con características xerofíticas que a uno correspondiente a la región biogeográfica de Yungas (Quiroga et al., en prep). Richardson (1998) reconoce el incremento del área forestada con especies exóticas de Eucaliptus y Pinus en Argentina durante el siglo XX. Asimismo, estudios recientes locales (Grau \& Aragón 2000, Grau et al. 2008) reconocen la capacidad invasora de Ligustrum lucidum, Morus alba, Gleditsia triacanthos y Psidium guajaba. Boose \& Holt (1999) exponen la facilidad de Arundo donax para invadir los márgenes fluviales, con marcados efectos en el consumo de agua y utilización de luz solar. La elevada cantidad de especies nativas en paralelo con la presencia de exóticas de los sitios ubicados sobre la Sierra de San Javier, podría indicar que estas últimas aún no han 
desplazado a las nativas. La relativa reciente creación de un área protegida en la zona podría haber posibilitado la recuperación de la vegetación ribereña nativa de los ríos Tafí, Las Cañitas y Muerto. El proceso de invasión por parte de las exóticas se encuentra íntimamente ligado con la intervención del ser humano (Richardson \& Pysek 2006). La presencia de especies foráneas dentro de la cuenca podría ser consecuencia remanente de los efectos de la actividad ganadera en el área, junto con la existencia actual de ganado a pequeña escala. Asimismo, los cambios en el uso del suelo (abandono de parcelas de cultivo) en las lomadas pedemontanas adyacentes a las riberas de los ríos Tafí y Las Cañitas, cambios que en algunos sitios ocurrieron en las propias riberas, podrían haber contribuido a la recuperación de la vegetación secundaria. Este último proceso condujo a la colonización de ambos sitios por especies nativas y exóticas, en diferente grado, según la posibilidad de llegada de los propágulos (Sirombra 2008).

La distribución de la vegetación se relacionó significativamente con ciertas características geomorfológicas. El mayor ancho del lecho seco de los sitios de ambas vertientes de la Sierra de San Javier se halla relacionado, por un lado, al menor orden de río, y por otro, al efecto de las actividades antropogénicas (obras de defensa, forestación de márgenes, extracción de áridos, ganadería, agricultura), factores que determinarían la formación de una masa vegetal distinta relacionada con las nuevas condiciones. Asimismo, un gradiente altitudinal se encuentra marcando diferencias en composición de especies entre sitios de la cuenca Río Lules. La accesibilidad a los sitios está determinada por este gradiente, factor que explica el mayor impacto antropogénico en sitios de menor altura tales como Pot y Tab. En relación con lo anterior, LJ1 y Anf. podrían ser considerados como sitios de referencia de vegetación ribereña de Yungas en virtud a la menor presión humana sobre el sistema.

Por otra parte, el ACD permitió identificar un grupo de especies comunes (Solanum riparium, Phenax laevigatus, Tipuana tipu,
Cestrum parqui, Carica quercifolia, Acacia macracantha, Celtis iguanaea, Juglans australis, Pisoniella arborescens, Baccharis salicifolia, Cinnamomum porphyrium y Eugenia uniflora), las que podrían ser consideradas como especies ribereñas siempre presentes en las zonas hidrográficas estudiadas.

Se encontró que la vegetación ribereña de la provincia biogeográfica de Yungas esta invadida por algunas especies exóticas, factor que actúa como amenaza para la composición florística natural de esta eco-región. El reconocimiento del rol que poseen los impactos antropogénicos en la propagación de exóticas y en la integridad de este ecosistema, vuelve urgente la necesidad de implementar medidas de control que garanticen el desarrollo de este tipo de actividad con mínimo impacto sobre la biodiversidad y la geomorfología. Por otra parte, este estudio detalló por primera vez la composición florística de las riberas de ríos subtropicales de montaña del NOA, identificó un grupo de especies comunes para las cuencas estudiadas y describió sitios de referencia de suma relevancia en estudios comparativos futuros. Estos resultados, constituyen información prioritaria para la aplicación de índices bióticos tales como el Q.B.R (Muneé et al. 1998) y el R.Q.I. (González del Tánago et al. 2006), diseñados para evaluar la calidad ecológica de las riberas fluviales, pero que deben ser adaptados previamente a las condiciones locales de ríos de Yungas. Asimismo, son necesarios para el diseño de un plan de gestión ambiental integral, aplicable a las riberas de los ríos del Noroeste Argentino.

\section{AGRADECIMIENTOS}

A Hugo R. Fernández por su colaboración incondicional para realizar este trabajo. A Fátima Romero y Marcela Correa por su ayuda en los muestreos y a Eduardo Domínguez por sus valiosos comentarios. A ILINOA, proyecto 26/ G446-1 y a la Fundación YPF. A la Agencia de Promoción Científica y Tecnológica, Pict: 01-12529, al Concejo de Investigación de la Universidad Nacional de Tucumán, 26-G309 y 
al Consejo Nacional de Investigación Científica y Tecnológica (CONICET).

\section{RESUMEN}

Se estudió la composición florística y distribución de los bosques ribereños de dos sistemas hidrográficos en una región subtropical andina. Utilizando técnicas uni y multivariadas, se probó la hipótesis que existe un bosque ribereño diferenciable, compuesto por vegetación nativa típica de la provincia fitogeográfica de Yungas, y que la distribución de la vegetación varió significativamente con las características geomorfológicas. Transectos paralelos a lo largo de los cursos de agua se utilizaron para recolectar los datos de presencia-ausencia de vegetación en once sitios. El Análisis de Correspondencia definió un grupo de especies ribereñas común para el área estudiada (Solanum riparium, Phenax laevigatus, Tipuana tipu, Cestrum parqui, Carica quercifolia, Acacia macracantha, Celtis iguanaea, Juglans australis, Pisoniella arborescens, Baccharis salicifolia, Cinnamomum porphyrium y Eugenia uniflora) e identificó dos sitios de referencia. La distribución de la vegetación ribereña varió significativamente con las características geomorfológicas a lo largo de los sitios estudiados. Los hábitats ribereños estuvieron compuestos por especies nativas y exóticas. Una flora ribereña distintiva, diferente en estructura y función a la vegetación terrestre adyacente, no pudo ser identificada. Especies ribereñas fueron similares a los estratos terrestres adyacentes. Estas especies no estarían limitadas por la proximidad al río. Impactos antropogénicos fueron factores importantes que regulan la introducción y el incremento de la vegetación exótica. La falta de regulación de algunas actividades en la zona podría causar problemas graves en la integridad de este ecosistema.

Palabras clave: Argentina, bosque de ribera, clima subtropical, ríos de montaña, Yungas.

\section{REFERENCIAS}

Barling, R.D. \& I.D. Moore. 1994. Role of buffer strips in management of waterway pollution: a review. Environ. Manage. 18: 543-558.

Boose, A.B. \& J.S. Holt. 1999. Environmental effects on asexual reproduction in Arundo donax. Weed Res. 39: $117-127$.

Brown, A.D. 2000. Development threats to biodiversity and opportunities for conservation in the mountain ranges of the upper Bermejo river basin, NW Argentina and SW Bolivia. Ambio 29: 445-449.
Ede F., \& T. Hunt. 2009. Understanding why weeds flourish in riparian zones. Thinking Bush-Thinking Weeds. Land \& Water Australia 8: 14-15.

González del Tánago, M., G. García de Jalón, F. Lara \& R. Garilleti. 2006. Índice RQI para la valoración de las riberas fluviales en el contexto de la directiva del marco de agua. Ingeniería Civil 143: 97-108.

Grau, H.R. \& R. Aragón. 2000. Árboles invasores de la Sierra de San Javier, Tucumán, Argentina, p. 5-20. In H. R. Grau \& R. Aragón (eds.). Ecología de árboles exóticos en las Yungas argentinas. Laboratorio de Investigaciones Ecológicas de las Yungas (LIEY)Universidad Nacional de Tucumán (UNT), Tucumán, Argentina.

Grau, H.R., M.E. Hernández, J. Gutierrez, N.I. Gasparri, M.C. Casavecchia, E.E. Flores \& L. Paolini. 2008. A peri-urban neotropical forest transition and its consequences for environmental services. Ecol. Soc. 13: 35 .

Gregory, S.V., F.J. Swanson, A.W. McKee \& K.W. Cummins. 1991. An ecosystem perspective of riparian zones. Bioscience 41: 540-551.

Hood, W.G. \& R.J. Naiman. 2000. Vulnerability of riparian zones to invasion by exotic vascular plants. Plant Ecol. 148: 105-114.

Lodge, D.M. 1993. Biological Invasions: Lessons for Ecology. Trends Ecol. Evol. 8: 133-137.

McCune, B. \& M.J. Mefford. 1999. Multivariate Analysis of Ecological Data.Version 4.25. MjM Software, Gleneden Beach, Oregon, EEUU.

Magurran A. 1989. Diversidad ecológica y su medición. University College of North Wales. Ediciones Vedra, Barcelona, España.

Manly, B.F.J. 1997. Randomization, Bootstrap and Monte Carlo Methods in Biology. Chapman \& Hall/CRC Press, Londres, UK.

Mesa, L.M. 2006. Morphometric analysis of a subtropical Andean basin (Tucumán, Argentina). Environ. Geol. 50: $1235-1242$.

Morales, J. M., M. Sirombra \& A. Brown. 1995. Riqueza de árboles en las Yungas argentinas, p. 163-174 In: A. D. Brown \& H. R. Grau (eds.). Investigación, Conservación y Desarrollo en Selvas Subtropicales de Montaña. Laboratorio de Investigaciones Ecológicas de las Yungas (LIEY)- Universidad Nacional de Tucumán (UNT), Tucumán, Argentina. 
Muneé A., C. Solá \& N. Prat. 1998. Q.B.R. Un índice rápido para la evaluación de la calidad de los ecosistemas de ribera. Tecnología del agua 175: 20-37.

Naiman, R.J., H. Décamps, J. Pastor \& C.A. Johnston. 1988. The potencial importance of boundaries to fluvial ecosystems. J. N. Am. Benthol. Soc. 7: 289-306.

Naiman, R.J., H. Décamps \& M. Pollock. 1993. The Role of Riparian Corridors in maintaining Regional Biodiversity. Ecol. Appl. 3: 209-212.

Naiman, R.J. \& H. Décamps. 1997. The ecology of interfaces: Riparian zones. Annu. Rev. Ecol. Syst. 28: 621-658.

Neiff, J. J. 2004. Bosques fluviales de la cuenca del Paraná. In: M.F. Arturi, J.L. Frangi \& J.F. Goya (eds.). Ecología y manejo de los bosques de Argentina. La Plata, Argentina.

Økland, R.H. 1996. Are ordination and constrained ordination alternative or complementary strategies in general ecological studies? J. Veg. Sci. 7: 289-292.

Parker, I.M. \& S.H. Reichard. 1997. Critical Issues in Invasion Biology for Conservation Science, p. 283-305. In P.L. Fiedler \& P.M. Kareiva (eds.). Conservation Biology for the Coming Decade. Editorial Chapman and Hall, Nueva York, EEUU.

Placci, L.G. 1995. Estructura y funcionamiento fenológico en relación a un gradiente hídrico en bosques del Este de Formosa. Tesis Doctoral. Universidad Nacional de La Plata, Argentina.

Prach, K. \& J. Straskrabová. 1996. Restoration of degraded meadows: an experimental approach, p. 87-93. In K. Prach, J. Jeník \& A.R.G. Large (eds.). Floodplain ecology and management. The Lunice River in the Trebon Biosphere Reserve, Central Europe SPB Academic Publishing, Amsterdam.
Risser, P.G. 1993. Ecotones. Ecol. Appli. 3: 369-445.

Richardson, D. 1998. Forestry trees as invasive aliens. Conserv. Biol. 12: 18-26.

Richardson D. \& P. Pysek. 2006. Plant invasions: merging the concepts of species invasiveness and community invasibility. Prog. Phys. Geog. 30: 409-431.

Richardson, D.M., P.M. Holmes, K.J. Esler, S.M. Galatowitsch, J.C. Stromber, S.P. Kirkman, P.

Pysek \& R.J. Hobbs. 2007. Riparian vegetation: degradation, alien plant invasions, and restoration prospects. Divers. Distrib. 13: 126-139.

Sirombra, M.G. 2008. Análisis temporal de cambios en el uso del suelo y su vinculación con procesos hidrogeomorfológicos en dos arroyos subtropicales de montaña. Tucumán, Argentina. Actas del IV Congreso Argentino de Limnología. Bariloche, Argentina.

Strahler, A.N. 1964. Quantitative geomorphology of drainage basin and channel networks, p. 4-76. In: V.T. Chow (ed.). Handbook of applied hydrology. McGraw Hill Book Co., Nueva York, EEUU.

Tang, S.M. \& D.R. Montgomery. 1995. Riparian buffers and potentially unstable ground. Environ. Manage. 19: 741-749.

Tausch, R.J., D.A. Charlet, D.A. Weixelman \& D.C. Zamudio. 1995. Patterns of ordination and classification instability resulting from changes in input data order. J. Veg. Sci. 6: 897-902.

\section{REFERENCIA DE INTERNET}

Zuloaga,F.O \& O. Morrone (eds.). 2009. Catálogo de Plantas Vasculares de la República Argentina. II. Instituto de Botánica Darwinion (Consultado: 16 de marzo 2009, http://www.darwin.edu.ar/publicaciones/catalogovascII/catalogovascII.asp 
ANEXO1

Listado de especies por familia, status y hábito, y abreviaciones utilizadas en el ACD

\section{APPENDIX 1}

List of species according to family, habit, status, and abbreviations used in DCA

\begin{tabular}{|c|c|c|c|c|}
\hline Especie & Familia & Status & Hábito & Código \\
\hline Schinus bumelioides Johnst. & Anacardiaceae & Nativa & Árbol & Sch \\
\hline Verbesina suncho (Griseb.) S.F. Blake & Asteraceae & Nativa & Arbusto & Ves \\
\hline Senecio peregrinus Griseb. & Asteraceae & Nativa & Arbusto & Sep \\
\hline Baccharis salicifolia (Ruiz et Pav.) Persoon & Asteraceae & Nativa & Arbusto & Bas \\
\hline Eupatorium lasiophtalmun Griseb. & Asteraceae & Nativa & Arbusto & Eul \\
\hline Tessaria integrifolia Ruiz et Pavon & Asteraceae & Nativa & Árbol & Tei \\
\hline Vernonia squamulosa Hook. et. Arn. & Asteraceae & Nativa & Arbusto & Vsq \\
\hline Vernonia fulta Griseb. & Asteraceae & Nativa & Apoyante & Vfu \\
\hline Jacaranda mimosifolia D.Don & Bignoniaceae & Nativa & Árbol & $\mathrm{Jac}$ \\
\hline Tabebuia avellanedae Lorentz ex Griseb. & Bignoniaceae & Nativa & Árbol & Tab \\
\hline Tecoma stans (L.) Juss. ex H. B. K. & Bignoniaceae & Nativa & Árbol & Tes \\
\hline Sambucus peruvianum H.B.K. & Caprifoliaceae & Nativa & Árbol & Sbp \\
\hline Carica quercifolia (St.Hil.) Solms-Laub. & Caricaceae & Nativa & Árbol & Car \\
\hline Celtis iguanaea (Jacq.) Sarg. & Celtidaceae & Nativa & Árbol & Cel \\
\hline Chamissoa altísima (Jacq.) H.B.K. & Chenopodiaceae & Nativa & Apoyante & Cha \\
\hline Terminalia triflora (Griseb.) Lillo & Combretaceae & Nativa & Árbol & Tet \\
\hline Ricinus communis $\mathrm{L}$. & Euphorbiaceae & Exótica & Arbusto & Ric \\
\hline Manihot grahamii Hook. & Euphorbiaceae & Nativa & Árbol & Man \\
\hline Acacia macracantha Humb. \& Bonpl. ex Willd. & Fabaceae & Nativa & Árbol & Amc \\
\hline Acacia praecox Griseb. & Fabaceae & Nativa & Árbol & Apr \\
\hline Tipuana tipu (Benth.) O. Kuntze & Fabaceae & Nativa & Árbol & Tit \\
\hline Parapiptadenia excelsa (Griseb.) Burkart & Fabaceae & Nativa & Árbol & $\mathrm{Pae}$ \\
\hline Acacia caven (Molina) Molina var. caven & Fabaceae & Nativa & Árbol & Acv \\
\hline $\begin{array}{l}\text { Anadenanthera colubrina (vell.) Bernan Ver (grises.). } \\
\text { atschul }\end{array}$ & Fabaceae & Nativa & Árbol & Ana \\
\hline Bauhinia candicans Benth. & Fabaceae & $?$ & Árbol & Bhc \\
\hline Cassia carnaval Speg. & Fabaceae & Nativa & Árbol & Cas \\
\hline Enterolobium contortisiliquum (Vell. Conc.) morong & Fabaceae & Nativa & Árbol & Eco \\
\hline Gleditsia triacanthos $\mathrm{L}$. & Fabaceae & Exótica & Árbol & Gtr \\
\hline Xylosma pubescens Griseb. & Flacourtiaceae & Nativa & Árbol & Xyl \\
\hline Juglans australis Griseb & Juglandaceae & Nativa & Árbol & Jua \\
\hline Cinnamomum porphyrium (Griseb.) Kosterm. & Lauraceae & Nativa & Árbol & Cin \\
\hline Persea americana Mill. & Lauraceae & Exótica & Árbol & Per \\
\hline Cederla lilloi C. DC. & Meliaceae & Nativa & Árbol & Ced \\
\hline Morus alba $\mathrm{L}$ & Moraceae & Exótica & Árbol & Mor \\
\hline Myrsine laetevirens (Mez) Arechav. & Myrsinaceae & Nativa & Árbol & Mir \\
\hline Myrcianthes pungens (Ver) Legrand & Myrtaceae & Nativa & Árbol & Myp \\
\hline Blepharocalyx salicifolius (H.B.K.) O.Berg. & Myrtaceae & Nativa & Árbol & Ble \\
\hline Eucalyptus grandis W Hill ex Maiden. & Myrtaceae & Exótica & Árbol & Euc \\
\hline Eugenia uniflora L. & Myrtaceae & Nativa & Árbol & Egu \\
\hline Myrcianthes mato (Griseb.) McVaugh & Myrtaceae & Nativa & Árbol & Mym \\
\hline Psidium guajava $\mathrm{L}$. & Myrtaceae & Exótica & Árbol & Psd \\
\hline Pisoniella arborescens var. glabrata Heimerl. & Nyctaginaceae & Nativa & Apoyante & Par \\
\hline
\end{tabular}


ANEXO1 (Continuación)

Listado de especies por familia, status y hábito, y abreviaciones utilizadas en el ACD

APPENDIX 1 (Continued)

List of species according to family, habit, status, and abbreviations used in DCA

\begin{tabular}{|c|c|c|c|c|}
\hline Especie & Familia & Status & Hábito & Código \\
\hline Pisonia ambigua Heimerl & Nyctaginaceae & Nativa & Árbol & Pam \\
\hline Ligustrum lucidum W.T. Aiton & Oleaceae & Exótica & Árbol & Lig \\
\hline Pinus taeda $\mathrm{L}$. & Pinaceae & Exótica & Árbol & Pin \\
\hline Piper hieronymi C. DC. & Piperaceae & Nativa & Arbusto & Pin \\
\hline Piper tucumanum C. DC. & Piperaceae & Nativa & Árbol & Pit \\
\hline Arundo donax L. & Poaceae & Exótica & Caña & Aru \\
\hline Guadua angustifolia Kunth & Poaceae & Exótica & Caña & Gua \\
\hline Ruprechtia laxiflora Meisn. & Polygonaceae & Nativa & Árbol & Rul \\
\hline Grevillea robusta A. Cunn. & Proteaceae & Exótica & Árbol & Gre \\
\hline Pyracantha angustifolia (Franch.) C.K. Schneid. & Rosaceae & Exótica & Arbusto & Cra \\
\hline Eriobotrya japonica (Thunb.) Lindl. & Rosaceae & Exótica & Árbol & Eri \\
\hline Prunus tucumanensis Lillo & Rosaceae & Nativa & Árbol & Pru \\
\hline Rubis imperialis Cham. \& Schltdl & Rosaceae & Nativa & Trepadora & Rub \\
\hline Psychotria carthagenensis Jacq. & Rubiaceae & Nativa & Arbusto & Psy \\
\hline Zanthoxilum fagara (L.) Sarg. & Rutacea & Nativa & Árbol & Fac \\
\hline Zanthoxilum naranjillo Griseb. & Rutacea & Nativa & Árbol & Fan \\
\hline Citrus aurantium $\mathrm{L}$. & Rutaceae & Exótica & Árbol & Cia \\
\hline Salix humboldtiana Willd. & Salicaceae & Nativa & Árbol & Sah \\
\hline Allophylus edulis (St. Hill.) Radlkofer & Sapindaceae & Nativa & Árbol & Alo \\
\hline Cupania vernalis Cambess. & Sapindaceae & Nativa & Árbol & Cup \\
\hline Solanum riparium Pers. Syn. & Solanaceae & Nativa & Árbol & Sol \\
\hline Cestrum parqui L’Hér. & Solanaceae & Nativa & Arbusto & Cep \\
\hline Cestrum strigillatum Ruiz \& Pav. & Solanaceae & Nativa & Arbusto & Ces \\
\hline Lycium cestroides Schldl. & Solanaceae & Nativa & Árbol & Lyc \\
\hline Heliocarpus popayanensis H. B. K. & Tiliaceae & Nativa & Árbol & Hel \\
\hline Trema micrantha (L.) Blume & Ulmaceae & Nativa & Árbol & Tmc \\
\hline Urera caracasana (Jacq.) Gaudich ex Griseb & Urticaceae & Nativa & Árbol & Urc \\
\hline Boehmeria caudata $\mathrm{Sw}$. & Urticaceae & Nativa & Arbusto & Boe \\
\hline Phenax laevigatus Wedd. & Urticaceae & Nativa & Arbusto & Phe \\
\hline Urera baccifera (L.) Gaud & Urticaceae & Nativa & Árbol & Urb \\
\hline Aloysia gratissima (Gill. ex Hook. et Arn.) Hicken & Verbenaceae & Nativa & Arbusto & Alg \\
\hline Duranta serratifolia (Griseb.) Kuntze & Verbenaceae & Nativa & Árbol & Dur \\
\hline Lantana camara $\mathrm{L}$. & Verbenaceae & Exótica & Arbusto & Lan \\
\hline
\end{tabular}

Los nombres científicos corresponden a los lineamientos establecidos en el Catálogo de Plantas Vasculares de Argentina (Zuloaga \& Morrone 2009).

Scientific names are according to the Catalogue of Vascular Plants of Argentina (Zuloaga \& Morrone 2009). 